\title{
Umami: An Alternative Japanese Approach to Reducing Sodium While Enhancing Taste Desirability
}

\author{
Shuhei Nomura1,2,3, Aya Ishizuka1,2, Shiori Tanaka1,3, Daisuke Yoneoka1,2,4, Hisayuki Uneyama5, \\ Kenji Shibuya ${ }^{1,6^{*}}$
}

${ }^{1}$ Department of Global Health Policy, Graduate School of Medicine, The University of Tokyo, Tokyo, Japan

${ }^{2}$ Department of Health Policy and Management, School of Medicine, Keio University, Tokyo, Japan

${ }^{3}$ Epidemiology and Prevention Group, Center for Public Health Sciences, National Cancer Center, Tokyo, Japan

${ }^{4}$ Graduate School of Public Health, St. Luke's International University, Tokyo, Japan

${ }^{5}$ Ajinomoto Co., Inc., Tokyo, Japan

${ }^{6}$ Soma COVID Vaccination Medical Center, Fukushima, Japan

Email: *kenjishibuya1@gmail.com

How to cite this paper: Nomura, S., Ishizuka, A., Tanaka, S., Yoneoka, D., Uneyama, H. and Shibuya, K. (2021) Umami: An Alternative Japanese Approach to Reducing Sodium While Enhancing Taste Desirability. Health, 13, 629-636.

https://doi.org/10.4236/health.2021.136047

Received: April 13, 2021

Accepted: June 1, 2021

Published: June 4, 2021

Copyright $\odot 2021$ by author(s) and Scientific Research Publishing Inc. This work is licensed under the Creative Commons Attribution International License (CC BY 4.0).

http://creativecommons.org/licenses/by/4.0/

\begin{abstract}
COVID-19 has highlighted the underlying global burden from non-communicable diseases (NCDs) and the lack of resiliency in health systems to tackle highly preventable risk factors, which have left populations vulnerable to acute health crises. The persistent issue of unhealthy behaviors, particularly those related to a high-sodium diet, suggests inadequate policy attention and insufficient funding for public health and behavioral research. This year, Japan will host the Tokyo Nutrition for Growth (N4G) Summit 2021. The summit is being held in the era of COVID-19, offering a historic opportunity to make a difference on the global health agenda for food and nutrition and to reaffirm the global commitment to prevent future health crises by addressing the growing burden of NCDs and building better prepared and more resilient health systems. This perspective article suggests that we, global health researchers and decision-makers, should rethink the value of using "Umami" substances as candidates for a practical salt-reducing alternative that originated in Japan (while maintaining the affordability, accessibility, availability, and desirability of healthy food). Reduced salt intake will help address the global syndemic of COVID-19 pandemic and high rates of hypertension, heart diseases, diabetes, and other NCDs. By addressing these issues, it will make countries more resilient to future pandemic threats.
\end{abstract}

\section{Keywords}

Umami, Sodium Reduction, Desirability, Eating Behavior 


\section{Introduction}

COVID-19, which is not only a pandemic but a "syndemic," has revealed the fundamental inequalities in the health security agenda [1]. It has also highlighted the underlying global burden from non-communicable diseases (NCDs) and a lack of resilient health systems to tackle highly preventable risk factors, which, in turn, have left populations vulnerable to acute health crises [2]. In 2013, World Health Organization (WHO) established the NCDs Global Monitoring Framework and set seven NCDs prevention goals [3]. Out of the seven goals, the only specific target for nutrients called on the member countries to aim for a $30 \%$ reduction in salt intake from 2011 by 2025. Since then, many salt reduction initiatives have been launched worldwide, accelerating the global salt reduction movement, but no country has achieved the $30 \%$ reduction goal so far [1]. The latest Global Burden of Disease Study 2019 (GBD) showed high salt intake as the top dietary risk contributing to the world's disease burden from NCDs [4], stressing the need for an urgent approach.

Japan has one of world's highest life expectancies [5], but a high-salt diet is the leading dietary risk factor for both mortality and morbidity [4] [6]. As Japan gears to host the Tokyo Nutrition for Growth (N4G) Summit 2021 later this year, the country has been presented with an opportune occasion to lead and make a difference on the global health agenda for food and nutrition. What's more, the summit is being held in the era of COVID-19, offering a historic opportunity to reaffirm the global commitment to prevent future health crises by addressing the growing burden of NCDs and building better-prepared and more resilient health systems. In this perspective article, using our previous research in Japan as an example, we present that there is a great deal of room for improving diet in order to maintain and further develop population health, while discussing why it is difficult to reduce salt intake. Then, we address a recent rise in the scientific understanding behind the salt-reducing potential of Umami, the fifth basic taste that enhances the flavor sensory and the salty taste. Finally, this article suggests that we, global health researchers and decision-makers, should consider the value of using "Umami" substances as candidates for a practical salt-reducing alternative. We believe that this paper contributes to the emerging literature on forestalling the escalating burden of a high-sodium diet.

\section{Japanese Diet and Dietary Risks}

In 2013, traditional Japanese cuisine was certified as an Intangible Cultural Heritage by the United Nations Educational, Scientific and Cultural Organization (UNESCO). In recent years, the low-caloric Japanese food has attracted attention from its likely link to healthy longevity. The traditional Japanese diet is characterized by a rice-based diet complemented by various plant-based ingredients and little animal source foods; some previous studies suggesting a possible association with long healthy life [7]. On the other hand, the Japanese diet also has dietary risks such as high salt, low whole grain, and low vegetables and fruits 
intakes. Like other countries, Japan has set these nutritional targets and is implementing policies to improve its population's eating habits.

We have been conducting research using Japanese National Health and Nutrition Survey data to estimate the current state of nutrition intake and forecast Japan's disease burden due to NCDs, assuming various future food intake scenarios up to 2040. As a result, we found that the percentage of people who meet the dietary goal for seven essential nutrients-designed to reduce the risk of lifestyle-related chronic diseases in Japan's 2015 national nutrient-based dietary guideline-was only $0.3 \%$ in 2017 [8]. We have also identified remarkable differences in disease burden between the scenarios, representing a wide scope of future trajectories of food intake with the potential for a tremendous decrease in the burden by 2040 [9] [10]. While it is important to note that foods are more than just a collection of nutrients and that focusing on individual nutrients does not necessarily translate to a healthy diet [11], there is room for Japan to improve its diet to maintain and further develop its population's health.

\section{Food Palatability/Satisfaction and Salt Intake}

In the course of our research, we found that vegetable and salt intakes are correlated. This correlation may result from the standard practice in many countries' food culture, including Japan, i.e., the vegetables are often cooked or seasoned with salt or other seasonings containing sodium [12] [13]. This correlation may send an alarming signal to the current nutritional trends in Europe and the United States, namely, the transition from animal-based proteins to plant-based proteins (protein transition). The shift from meat-based proteins to plant-based also means a change to low-saturated fats (and high-vitamins and high-minerals), which relates to the palatability of the food and the satisfaction in eating the food. This transition may increase consumption of food or other tastes to compensate for the lack of palatability and satisfaction in a low-fat diet. The simplest or most accessible way to enhance the food-consuming experience is by adding salt. This dependence on salt for palatability has been shown in food cultures around the world. It is also shown in a nutritional survey that analyzed 290 savory dishes offered at 45 plant-based restaurants in 2020 in the United Kingdom. The amount of salt in foods offered at these restaurants was substantially higher than that of ordinary restaurants in the United Kingdom [14]. In another survey, the salt content of more than 150 meat substitute products, including meat-free burgers, sausages, and mincemeat, was investigated, and the results showed that meat-free burgers contained more salt per serving than beef burgers [15].

Why is salt reduction so difficult? Salt has many advantages in our diet, such as enhancing flavor, modifying textures, and preserving foods. Sodium chloride $(\mathrm{NaCl})$ is the major dietary nutrient that activates the sensation of saltiness among the five basic tastes, which are the essential sensory inputs to the brain as markers of food selection behaviors in human life. We cannot live without $\mathrm{NaCl}$, just as our body fluids contain around $140 \mathrm{mEq} / \mathrm{L}$ of sodium. Therefore, our 
brain is wired to trigger a sense of discomfort if there is a lack of $\mathrm{NaCl}$ in the body. In contrast, ingestion of $\mathrm{NaCl}$ leads to feelings of pleasure and enjoyment, such as palatability and a sense of satisfaction gained from eating. Reducing salt is naturally very difficult, as it would be a counterintuitive act to our natural desire for a savory taste.

Dietary habits, which are confounded by our taste, custom, and culture, are very difficult to modify. In today's world, where the cheapest table salt and savory foods rich in salt are already abundant around us, a "low-salt diet lacking palatability" becomes a significant hurdle in our quest for healthy dietary behaviors. In addition to the knowledge that salt reduction is necessary to maintain our health, we should provide the general public with specific cooking methods and flavorful, low-salt food products to keep the meals' palatability and satisfaction. To do so, we believe that flavoring technology is one of the most promising solutions.

\section{Umami: A Taste from Japan}

In 1908, Umami, or savoriness, was discovered by a Japanese chemist, Dr. Kikunae Ikeda, as the fifth basic taste uniquely different from the classic four basic tastes, such as saltiness, sweetness, bitterness, and sourness. Dr Ikeda identified monosodium glutamate (MSG, one of the most abundant naturally occurring amino acids) as a key component that gives Umami flavor in kombu stock, a traditional Japanese soup stock [16]. After its discovery, Umami-causing substances, including MSG, became the driving force within the amino acid fermentation industry in Japan; now, more than 3.5 million tons are consumed annually worldwide, contributing to the palatability of foods in each food culture. Umami is a basic taste that enhances the flavor sensory and the salty taste. Since the tasteenhancing effect of MSG on low-salt soup was recognized [17], food scientists verified that Umami substances enhance the savory flavor and palatability of lowsalt diets.

However, getting a global recognition of MSG as an effective and practical solution for salt reduction is still a huge challenge. For decades, MSG has been linked to various health problems, such as headaches and allergic reactions known as the "Chinese restaurant syndrome". A previous report published in 1968 suggested that eating Chinese food caused numbness and palpitations in the neck and arms because of MSG in the food [18]. Although the report did not provide any statistical data, news about the syndrome has spread worldwide. Some studies have also shown that MSG intake is associated with other symptoms such as asthma, urticaria, atopic dermatitis, respiratory difficulty, and tachycardia [19] [20]. However, studies, including double-blind placebo-controlled trials, confirmed that the relationship between MSG intake and the development of the symptoms did not have a plausible association, and that those who self-identified as sensitive to MSG did not show reproducibility of the different symptoms reported [21] [22]. These observations, along with the limitation of not being able to assume the existence of a positive association shown in some studies, indicate 
that MSG intake is unlikely to result in the development of symptoms associated with Chinese restaurant syndrome [23]. In addition, there is no strong scientific evidence to suggest that MSG is linked to the development of asthma, urticaria, angioedema or rhinitis [24]. The safety of MSG has also been evaluated by scientific committees and regulatory agencies, including the Joint FAO/WHO Expert Committee on Food Additives (JECFA), the Scientific Committee on Food (SCF) of the European Commission, and the United States Food and Drug Administration (FDA). These organizations came to similar conclusions about the safety of MSG, agreeing that MSG is safe at levels normally consumed by the general public, and that there is no evidence linking current use of MSG foods to serious long-term medical problems in the general public [25].

Recently, academic institutions such as the Institute of Medicine in the United States have listed Umami substances as candidates for a practical salt-reducing alternative [26]. Wallace et al. (2019) estimated that incorporating Umami substances into a savory seasoning of processed foods in the United States could reduce sodium intake by at least $3 \%-8 \%$ for the country's population [27]. The source of salt intake, however, is highly dependent on the dietary habits and cooking process in each country [28]. Processed foods, including ultra-processed foods, are the primary sources of salt intake for countries like the United States and the United Kingdom, whereas seasonings (table salt, soy sauce, etc.) and home cooking are the primary sources for countries like Japan, Italy, and China. The permeation of Umami taste also varies depending on the food culture, and the results of the United States cannot be easily generalizable to other countries. Therefore, it would be worth assessing the possibility of reducing salt intake at the national and regional levels through the use of Umami substances in different countries and regions.

\section{Conclusions-Reducing Sodium While Enhancing Desirability Using Umami Taste}

In 2020, the Global Panel on Agriculture and Food Systems for Nutrition added a new dimension, "desirability," to the existing 3A (affordability, accessibility, availability) for achieving a sustainable and healthy diet [29]. Palatability is the most fundamental desirability in a sustainable healthy diet, especially for a lowsalt diet, and MSG is a potent palatability enhancer. Now is the time for us to consider the potential of using Umami taste and substances as a healthy and natural solution for salt reduction, which will also help alleviate the global burden from hypertension, heart diseases, diabetes, and other NCDs.

\section{Author Contribution}

S.N., H.U., and K.S. conceptualized and drafted the original manuscript, which was revied and edited by S.T., D.Y. and K.S.

\section{Funding Statement}

This article is partially supported by a joint research grant from Ajinomoto Co., 
Inc. H.U. is employed by the commercial founder, Ajinomoto Co., Inc. The commercial funder provided support in the form of salaries for H.U., but did not have any additional role in the decision to publish or the preparation of this manuscript.

\section{Conflicts of Interest}

S.N. and K.S. report a grant from the Ajinomoto Co., Inc. H.U. declares that he is employed by Ajinomoto Co., Inc. and has no other competing interests. All other authors declare no competing interests.

\section{References}

[1] Development Initiatives (2020) Global Nutrition Report: Action on Equity to End Malnutrition. Development Initiatives, Bristol.

[2] Belanger, M.J., Hill, M.A., Angelidi, A.M., et al. (2020) Covid-19 and Disparities in Nutrition and Obesity. New England Journal of Medicine, 383, e69. https://doi.org/10.1056/NEJMp2021264

[3] World Health Organization (2011) NCD Global Monitoring Framework. https://www.who.int/publications/i/item/ncd-surveillance-global-monitoring-frame work

[4] GBD 2019 Risk Factors Collaborators (2020) Global Burden of 87 Risk Factors in 204 Countries and Territories, 1990-2019: A Systematic Analysis for the Global Burden of Disease Study 2019. The Lancet, 396, 1223-1249.

[5] GBD 2019 Demographics Collaborators (2020) Global Age-Sex-Specific Fertility, Mortality, Healthy Life Expectancy (HALE), and Population Estimates in 204 Countries and Territories, 1950-2019: A Comprehensive Demographic Analysis for the Global Burden of Disease Study 2019. The Lancet, 396, 1160-1203.

[6] Nomura, S., Sakamoto, H., Glenn, S., et al. (2017) Population Health and Regional Variations of Disease Burden in Japan, 1990-2015: A Systematic Subnational Analysis for the Global Burden of Disease Study 2015. The Lancet, 390, 1521-1538. https://doi.org/10.1016/S0140-6736(17)31544-1

[7] Imai, T., Miyamoto, K., Sezaki, A., et al. (2019) Traditional Japanese Diet ScoreAssociation with Obesity, Incidence of Ischemic Heart Disease, and Healthy Life Expectancy in a Global Comparative Study. The Journal of Nutrition, Health and Aging, 23, 717-724. https://doi.org/10.1007/s12603-019-1219-5

[8] Yoneoka, D., Nomura, S., Kurotani, K., et al. (2019) Does Japan's National Nutrient-Based Dietary Guideline Improve Lifestyle-Related Disease Outcomes? A Retrospective Observational Cross-Sectional Study. PLoS ONE, 14, e0224042. https://doi.org/10.1371/journal.pone.0224042

[9] Nomura, S., Yoneoka, D., Tanaka, S., et al. (2020) Forecasting Disability-Adjusted Life Years for Chronic Diseases: Reference and Alternative Scenarios of Salt Intake for 2017-2040 in Japan. BMC Public Health, 20, 1475. https://doi.org/10.1186/s12889-020-09596-3

[10] Yoneoka, D., Nomura, S., Tanaka, S., et al. (2020) Prediction of Disability-Adjusted Life Years for Diseases Due to Low Fruit Intake 2017-2040 in Japan. Public Health Nutrition, 1-21. https://doi.org/10.1017/S1368980020004541

[11] Jacobs, D.R., Gross, M.D. and Tapsell, L.C. (2009) Food Synergy: An Operational Concept for Understanding Nutrition. The American Journal of Clinical Nutrition, 89, 1543S-1548S. https://doi.org/10.3945/ajcn.2009.26736B 
[12] Ahern, S.M., Caton, S.J., Bouhlal, S., et al. (2013) Eating a Rainbow. Introducing Vegetables in the First Years of Life in 3 European Countries. Appetite, 71, 48-56. https://doi.org/10.1016/j.appet.2013.07.005

[13] Feng, Y., Albiol Tapia, M., Okada, K., et al. (2018) Consumer Acceptance Comparison between Seasoned and Unseasoned Vegetables. Journal of Food Science, 83, 446-453. https://doi.org/10.1111/1750-3841.14027

[14] Action on Salt (2020) Salt Content of Vegan and Plant-Based Meals Served in the Out of Home Sector.

http://www.actiononsalt.org.uk/salt-surveys/2020/salt-awareness-week-plant-basedmeals-in-the-out-of-home-sector

[15] Action on Salt (2018) Meat Alternatives Survey 2018. http://www.actiononsalt.org.uk/salt-surveys/2018/meat-alternatives-survey

[16] Beauchamp, G.K. (2009) Sensory and Receptor Responses to Umami: An Overview of Pioneering Work. American Journal of Clinical Nutrition, 90, 723S-727S. https://doi.org/10.3945/ajcn.2009.27462E

[17] Yamaguchi, S. and Takahashi, C. (1984) Interactions of Monosodium Glutamate and Sodium Chloride on Saltiness and Palatability of a Clear Soup. Journal of Food Science, 49, 82-85. https://doi.org/10.1111/j.1365-2621.1984.tb13675.x

[18] Kwok, R.H. (1968) Chinese-Restaurant Syndrome. New England Journal of Medicine, 278, 796. https://doi.org/10.1056/NEJM196804042781419

[19] Gann, D. (1977) Ventricular Tachycardia in a Patient with the "Chinese Restaurant Syndrome". Southern Medical Journal, 70, 879-881. https://doi.org/10.1097/00007611-197707000-00036

[20] Ratner, D., Eshel, E. and Shoshani, E. (1984) Adverse Effects of Monosodium Glutamate: A Diagnostic Problem. Israel Journal of Medical Sciences, 20, 252-253.

[21] Yang, W.H., Drouin, M.A., Herbert, M., et al. (1997) The Monosodium Glutamate Symptom Complex: Assessment in a Double-Blind, Placebo-Controlled, Randomized Study. The Journal of Allergy and Clinical Immunology, 99, 757-762. https://doi.org/10.1016/S0091-6749(97)80008-5

[22] Geha, R.S., Beiser, A., Ren, C., et al. (2000) Multicenter, Double-Blind, PlaceboControlled, Multiple-Challenge Evaluation of Reported Reactions to Monosodium Glutamate. The Journal of Allergy and Clinical Immunology, 106, 973-980. https://doi.org/10.1067/mai.2000.110794

[23] Maluly, H.D.B., Arisseto-Bragotto, A.P. and Reyes, F.G.R. (2017) Monosodium Glutamate as a Tool to Reduce Sodium in Foodstuffs: Technological and Safety Aspects. Food Science \& Nutrition, 5, 1039-1048. https://doi.org/10.1002/fsn3.499

[24] Williams, A.N. and Woessner, K.M. (2009) Monosodium Glutamate "Allergy": Menace or Myth? Clinical \& Experimental Allergy, 39, 640-646.

https://doi.org/10.1111/j.1365-2222.2009.03221.x

[25] Walker, R. and Lupien, J.R. (2000) The Safety Evaluation of Monosodium Glutamate. Journal of Nutrition, 130, 1049s-1052s. https://doi.org/10.1093/jn/130.4.1049S

[26] Institute of Medicine (2010) Strategies to Reduce Sodium Intake in the United States. The National Academies Press, Washington DC.

[27] Wallace, T.C., Cowan, A.E. and Bailey, R.L. (2019) Current Sodium Intakes in the United States and the Modelling of Glutamate's Incorporation into Select Savory Products. Nutrients, 11, 2691. https://doi.org/10.3390/nu11112691

[28] Anderson, C.A., Appel, L.J., Okuda, N., et al. (2010) Dietary Sources of Sodium in 
China, Japan, the United Kingdom, and the United States, Women and Men Aged 40 to 59 Years: The INTERMAP Study. Journal of the American Dietetic Association, 110, 736-745. https://doi.org/10.1016/j.jada.2010.02.007

[29] Global Panel on Agriculture and Food Systems for Nutrition (2020) Future Food Systems: For People, Our Planet, and Prosperity. Global Panel on Agriculture and Food Systems for Nutrition, Geneva. 